\title{
Modified ovarian response prediction index: a novel index for ovarian response prediction in GnRH agonist cycles
}

\section{B. Kalpana*, Soumya Ranjan Panda}

Department of Reproductive Medicine, Guru Hospital, Madurai, Tamil Nadu, India

Received: 19 April 2019

Accepted: 30 May 2019

\section{* Correspondence:}

Dr. B. Kalpana,

E-mail: balamurugan_kalpana@rediffmail.com

Copyright: $(\subset)$ the author(s), publisher and licensee Medip Academy. This is an open-access article distributed under the terms of the Creative Commons Attribution Non-Commercial License, which permits unrestricted non-commercial use, distribution, and reproduction in any medium, provided the original work is properly cited.

\begin{abstract}
Background: Evaluation of the ovarian reserve is necessary to achieve an appropriate controlled ovarian stimulation (COS). This can be done by correctly predicting the ovarian response. The objective of this study was to derive a simple index by combining the above parameters which will be helpful determining ovarian response.

Methods: This retrospective analysis was performed at Guru hospital, Madurai, involving 162 patients between July 2016 and July 2018. Inclusion criteria was all patients attending for their first ICSI (intracytoplasmic sperm injection) cycle between the above period, GnRH agonist protocol as the method of ovarian stimulation, no history of any previous ovarian surgery, presence of both ovaries and no evidence of any obvious endocrine disorders. We calculated MORPI values by multiplying the AMH ( $\mathrm{ng} / \mathrm{ml})$ level by the number of antral follicles $(2-9 \mathrm{~mm})$, and the result was divided by the age (years) of the patient and the day- 3 serum FSH level.

Results: At a cut-off value of 35 (AUC-0.952) for collection of $\geq 4$ oocytes and 140 (AUC-0.952) for collection of $\geq$ 15 oocytes, MORPI was found to have optimum sensitivity and specificity under ROC curve analysis.

Conclusions: MORPI is a simple, precise and cost effective index to predict a low ovarian response, the collection of $>4$ MII oocytes and an excessive ovarian response in infertile women. This index also has a good ability to predict the clinical pregnancy rate. This might be used to improve the cost-benefit ratio of ovarian stimulation regimens.
\end{abstract}

Keywords: Antral follicle count, Anti-Müllerianhormone, Controlled ovarian stimulation, Follicle-stimulating hormone, Modified ovarian response prediction index

\section{INTRODUCTION}

The size of the primordial follicle pool as well as the oocyte quality, constitute the ovarian reserve for a woman. ${ }^{1,2}$ Ovarian reserve is the primary factor for determining ovarian sensitivity in cycles of controlled ovarian stimulation (COS). In other words evaluation of the ovarian reserve is necessary to achieve an appropriate controlled ovarian stimulation (COS). Hence the correct optimization and individualization of the gonadotropin start dose is an extremely important clinicaldecision in IVF cycles. This can be done by correctly predicting the ovarian response. There are various biomarkers to measure ovarian reserve, including antiMüllerianhormone (AMH), day-3 follicle-stimulating hormone (FSH), and antral follicle count (AFC), each with its own pros and cons. The objective of this study was to derive a simple index by combining the above parameters which will be helpful determining ovarian response. We also compared the simple index derived by us, modified ovarian response prediction index (MORPI) with another recently developed simple index the ovarian response prediction index (ORPI) developed by Oliveira et al. ${ }^{3}$

\section{METHODS}

This retrospective analysis was performed at Guru hospital, Madurai, involving 162 patients between July 2016 and July 2018. The flow chart showing strategy of 
patient selection has been shown in Figure 1. Data regarding the age of the patient, BMI, serum levels of $\mathrm{AMH}, \mathrm{FSH}$, antral follicle count etc. were collected from previous hospital records. Inclusion criteria was all patients attending for their first ICSI (intracytoplasmic sperm injection) cycle between the above period, $\mathrm{GnRH}$ agonist protocol as the method of ovarian stimulation, no history of any previous ovarian surgery, presence of both ovaries and no evidence of any obvious endocrine disorders. The exclusion criteria were the presence of ovarian cysts as assessed by transvaginal ultrasound, previous history of ovarian surgery and patients who have attended menopause.

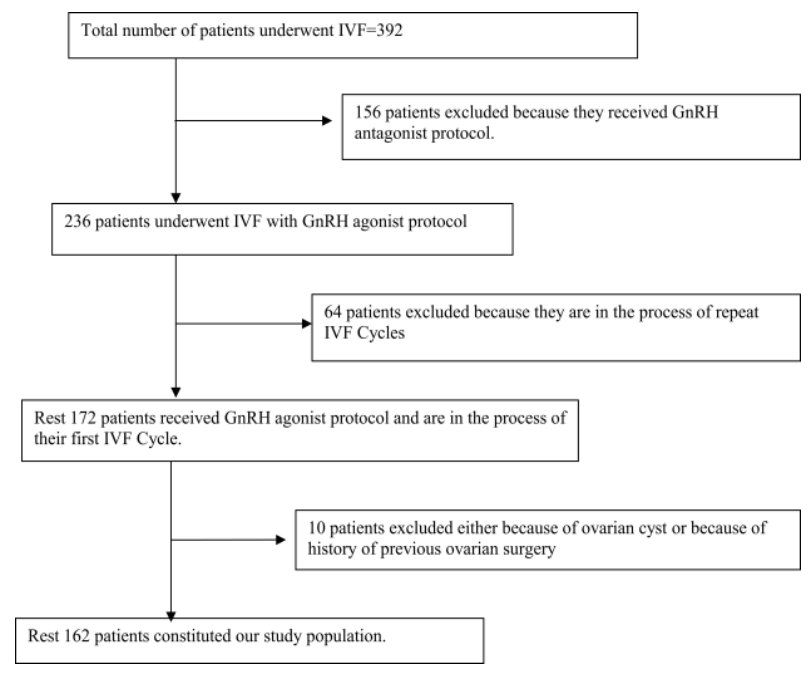

Figure 1: Patient selection.

\section{Ovarian stimulation protocol}

The pituitary down regulation began during the luteal phase of the previous menstrual cycle with the GnRH-a, leuprolide acetate at a dose of $1 \mathrm{mg} /$ day for 14 days. The ovaries were then stimulated with a fixed dose of 150$225 \mathrm{IU}$ of hMG for a period of 7 days. On day 8 of the ovarian stimulation, the follicular development was monitored by a transvaginal ultrasound and accordingly the dose modified. A senior IVF consultant was involved in all the clinical decisions.

\section{Calculation of ovarian response prediction index (ORPI) and modified ovarian response prediction index (MORPI)}

Initially the ORPI (ovarian response prediction index) was developed by Oliveira et al. $^{3}$

They calculated ORPI values by multiplying the AMH ( $\mathrm{ng} / \mathrm{ml})$ level by the number of antral follicles $(2-9 \mathrm{~mm})$, and the result was divided by the age (years) of the patient. This definition of ORPI was based on previous evaluations that found that the ovarian response to stimulation had positive correlations with the AMH levels and number of antral follicles and was negatively correlated with the patient's age. But in this definition of
ORPI, the serum FSH which is supposed to correlate inversely with ovarian reserve is not considered. This fact encouraged us to develop a new ovarian response index known as modified ovarian response prediction index (MORPI) in which the serum FSH level of patient is also taken into consideration.

\section{The MORPI can be defined by the following equation}

$$
\text { MORPI }=\frac{(A M H \times A F C)}{(\text { Patient age } \times \text { FSH })}
$$

The factor 1000 was multiplied in the above equation in order to negate for the numbers beyond decimals (so that we can find the result in whole numbers instead of small fractions). The primary endpoints were the total number of oocytes and the number of metaphase II (MII) oocytes retrieved. The secondary endpoints were the number of follicles $\geq 10 \mathrm{~mm}, \geq 16 \mathrm{~mm}$ and $\geq 18 \mathrm{~mm}$ on the day of HCG administration and clinical pregnancy.

\section{Statistical analysis}

Receiver operating characteristic (ROC) curves were constructed to examine the performance of the MORPI and ORPI in predicting the retrieval of $\geq 4$ oocytes, $\geq 4 \mathrm{MII}$ oocytes and $\geq 15$ oocytes and clinical pregnancy. An optimized cut-off point was obtained for each. The discriminative performance was assessed by the area under the curve (AUC) of the ROC curve. All the statistical analysis was performed using statistical software SPSS version 20.

\section{RESULTS}

The general characteristics of the study population are summarized in Table 1.

Table 1: Demographic parameters of included patients.

\begin{tabular}{|ll|}
\hline Parameters & Total study population \\
\hline Age & $31.45 \pm 4.68$ \\
\hline AMH & $2.54 \pm 1.91$ \\
\hline AFC & $11.41 \pm 4.50$ \\
\hline Day-2 FSH & $7.23 \pm 1.61$ \\
\hline ORPI & $1.15 \pm 1.23$ \\
\hline MORPI & $179.07 \pm 203.3$ \\
\hline Follicles on hCG day & \\
\hline$\geq 10$ mm & $12.42 \pm 3.36$ \\
\hline$\geq 16$ mm & $5.70 \pm 1.53$ \\
\hline$\geq 18$ mm & $3.69 \pm 1.11$ \\
\hline Retrieved Oocytes & \\
\hline Total & $10.78 \pm 6.11$ \\
\hline Metaphase -II & $6.88 \pm 4.45$ \\
\hline Metaphase - I & $2.84 \pm 1.61$ \\
\hline Germinal vesicle & $0.98 \pm 0.77$ \\
\hline Clinical pregnancy rate & $80 / 162(49.3 \%)$ \\
\hline
\end{tabular}


Of all 162 women, the mean age was $31.64 \pm 4.68$ years (range 22-45), the mean AMH level was $2.54 \pm 1.91 \mathrm{ng} / \mathrm{mL}$ (range 0.01-9.4), the mean AFC was 11.41 \pm 4.5 (range 428) and the mean serum $\mathrm{FSH}($ day-2) was 7.23 \pm 1.61 (range 4-12.4). The mean MORPI was 179.07 \pm 203.33 (range 0.08-595.68) and the mean ORPI was $1.15 \pm 1.23$ (range 0.001-7.1). On regression analysis (Table 2) both MORPI and ORPI demonstrated significant $(\mathrm{P}<0.05)$ positive correlations with total number of oocytes collected $(\mathrm{r}=0.8$ for MORPI versus
0.787 for ORPI) and total number of MII oocytes $(\mathrm{r}=0.801$ for MORPI versus 0.792 for ORPI).

Additionally, all the other markers of ovarian response showed statistically significant correlations with the variables analysed. However, the association provided by the MORPI and ORPI improved the correlation because the individual correlation coefficients of each marker of ovarian response (age, AMH and AFC) were always lower than that presented by the MORPI.

Table 2: Regression analysis.

\begin{tabular}{|llll|}
\hline \multicolumn{2}{|l|}{ Correlation coefifient $(r)$} & $R-$ square & p-Value \\
\hline Age & & & $<.449$ \\
\hline AMH & -0.670 & 0.585 & $<0.001$ \\
\hline FSH & +0.765 & 0.389 & $<0.001$ \\
\hline AFC & -0.623 & 0.595 & $<0.001$ \\
\hline ORPI & +0.771 & 0.619 & $<0.001$ \\
\hline MORPI & +0.787 & 0.640 & $<0.001$ \\
\hline Total no. of M-II oocytes collected & +0.800 & & $<0.001$ \\
\hline Age & -0.668 & 0.446 & $<0.001$ \\
\hline AMH & +0.783 & 0.614 & $<0.001$ \\
\hline FSH & -0.587 & 0.344 & $<0.001$ \\
\hline AFC & +0.729 & 0.532 & $<0.001$ \\
\hline ORPI & +0.792 & 0.628 & $<0.001$ \\
\hline MORPI & +0.801 & 0.642 & $<0.001$ \\
\hline
\end{tabular}

Table 3: ROC curve analysis.

\begin{tabular}{|c|c|c|c|c|c|c|}
\hline & AUC & CUT & Sensitivity (\%) & Specificity (\%) & $\begin{array}{l}\text { Positive } \\
\text { predictive value }\end{array}$ & $\begin{array}{l}\text { Negative predictive } \\
\text { value }\end{array}$ \\
\hline \multicolumn{7}{|c|}{ ROC curve analysis for retrieval of $\geq 4$ total oocytes } \\
\hline MORPI & 0.952 & 35 & 88 & 93 & 76 & 96 \\
\hline ORPI & 0.921 & 0.25 & 88 & 89 & 69 & 96 \\
\hline AMH & 0.890 & 1.4 & 85 & 85 & 60 & 95 \\
\hline AFC & 0.913 & 10 & 97 & 72 & 48 & 98 \\
\hline \multicolumn{7}{|c|}{ ROC curve analysis for retrieval of $\geq 4$ M-II oocytes } \\
\hline MORPI & 0.953 & 108.33 & 94 & 84 & 77 & 96 \\
\hline ORPI & 0.943 & 0.475 & 80 & 96 & 92 & 90 \\
\hline AMH & 0.909 & 1.69 & 82 & 90 & 82 & 90 \\
\hline $\mathrm{AFC}$ & 0.896 & 10 & 86 & 81 & 72 & 91 \\
\hline \multicolumn{7}{|c|}{ ROC curve analysis for retrieval of $\geq 15$ total oocytes } \\
\hline MORPI & 0.918 & 140 & 85 & 88 & 94 & 73 \\
\hline ORPI & 0.928 & 0.95 & 92 & 90 & 95 & 83 \\
\hline AMH & 0.816 & 2.78 & 92 & 56 & 82 & 75 \\
\hline $\mathrm{AFC}$ & 0.868 & 15 & 92 & 48 & 79 & 72 \\
\hline \multicolumn{7}{|c|}{ ROC curve analysis for clinical pregnancy rate } \\
\hline MORPI & 0.708 & 63.5 & 45 & 85 & 75 & 60 \\
\hline ORPI & 0.702 & 0.365 & 42 & 86 & 76 & 59 \\
\hline AMH & 0.681 & 1.5 & 47 & 81 & 72 & 60 \\
\hline AFC & 0.685 & 7 & 39 & 95 & 88 & 60 \\
\hline
\end{tabular}




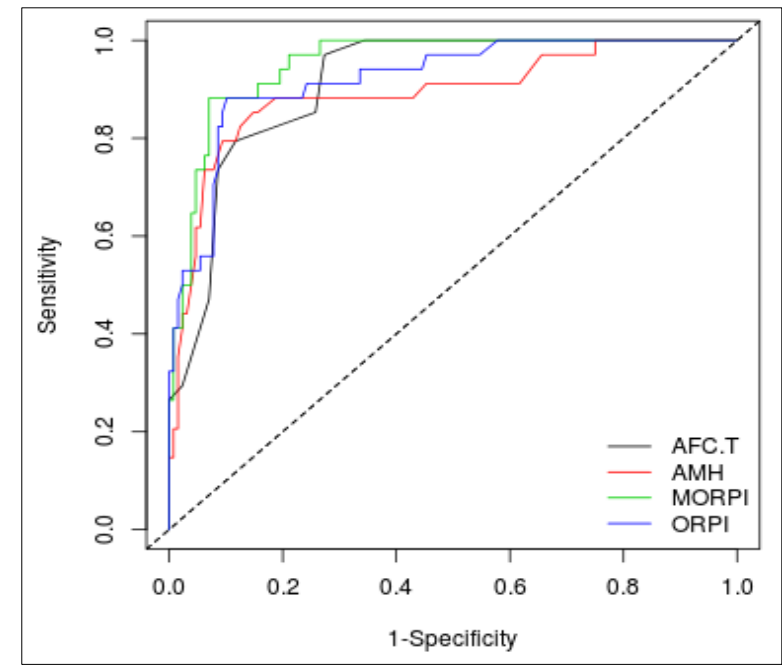

Figure 2: ROC curve analysis for retrieval of $\geq 4$ total oocytes.

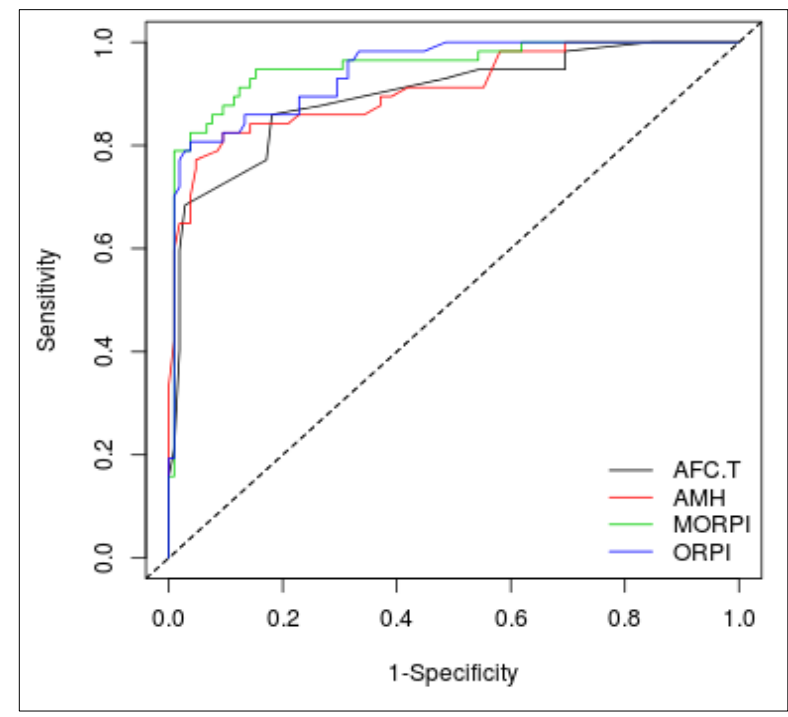

Figure 3: ROC curve analysis for retrieval of $\geq 4$ M-II oocytes.

The performance of the MORPI and ORPI as a prognostic test was observed using ROC curves (Table $3)$. Regarding the probability of collecting $\geq 4$ oocytes (Table 3, Figure 2), the ROC curve showed an area under the curve of 0.952 , indicating that the MORPI had an excellent prognostic potency for this point. At the cut-off threshold of 35, it showed the optimal compromise between specificity (93\%) and sensitivity (88\%) and between positive predictive value $(76 \%)$ and negative predictive value (96\%). Similarly for ORPI the ROC curve showed an area under the curve of 0.921. At the cut-off threshold of 0.25 , it showed the optimal compromise between specificity (89\%) and sensitivity (88\%) and between positive predictive value $(69 \%)$ and negative predictive value $(96 \%)$. The ROC curves also revealed good prognostic potency for all other factors (AMH and AFC) analysed. However, the AUC presented by the MORPI was always higher than those presented by all others factors.

Similarly, in regards to the probability of collecting $\geq$ 4MII oocytes (Table 3 and Figure 3), the ROC curve had an area under the curve of 0.953 , for MORPI and 0.943 for ORPI indicating that both indices had an excellent prognostic potency for this issue. Setting the threshold at 108.33 for MORPI and at 0.475 for ORPI, we could obtain maximum sensitivity and specificity for each. The ROC curves also revealed good prognostic potency for all other factors (age, AMH and AFC) analysed. However, the AUC presented by the MORPI was always higher than those presented by all others.

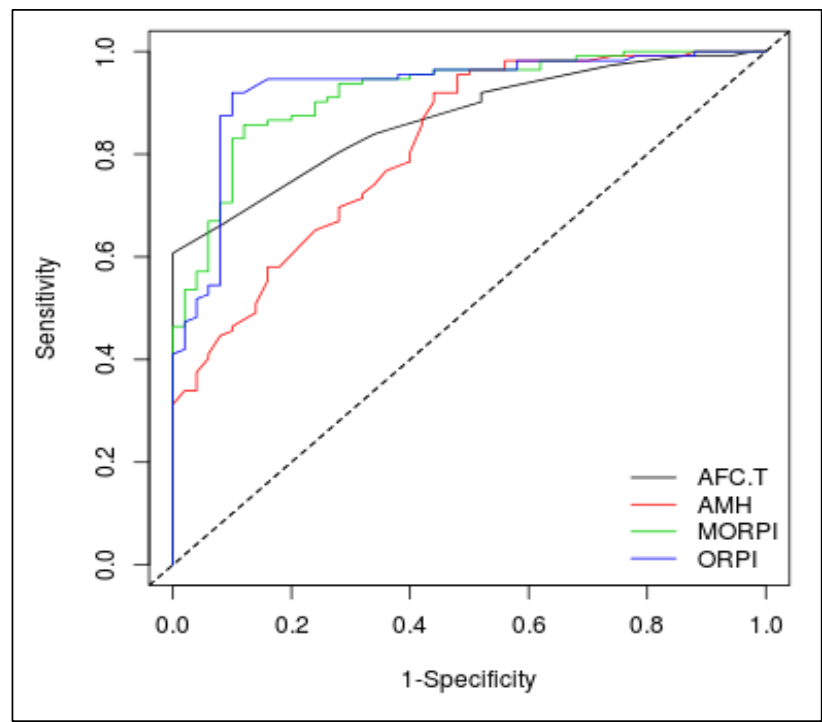

Figure 4: ROC curve analysis for retrieval of $\geq 15$ total oocytes.

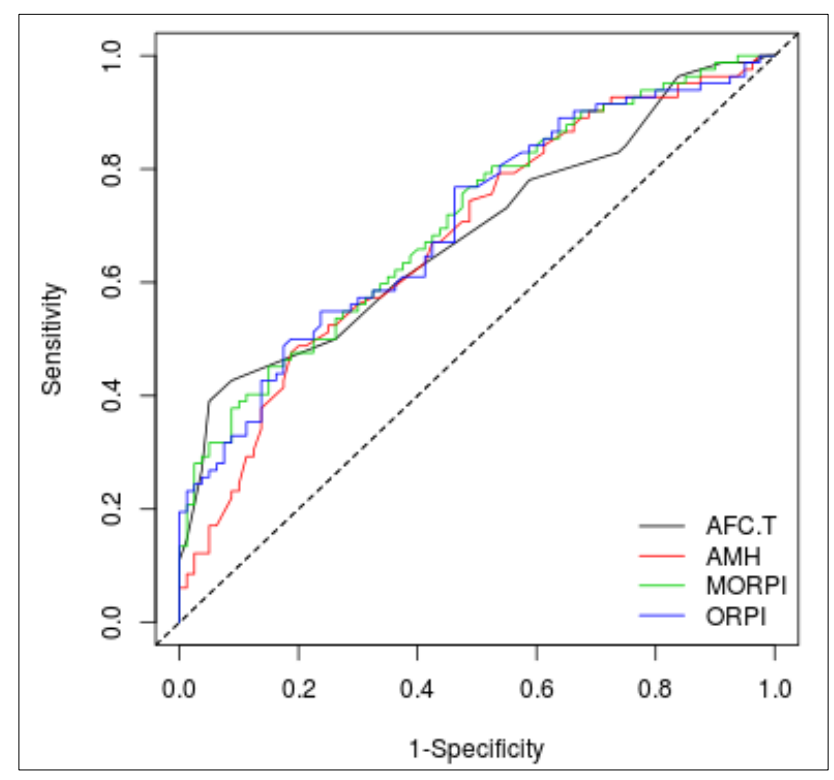

Figure 5: ROC curve analysis for clinical pregnancy rate. 
In the same way, the ROC curve for the probability of collecting $\geq 15$ oocytes (Table 3 and Figure 4) gave an area under the curve of 0.918 for MORPI while it was 0.928 for ORPI indicating that although both MORPI and ORPI have an excellent prognostic potency, ORPI seems to have little better performance than MORPI for this issue. This could be due to variation of correlation potency of serum FSH with ovarian reserve. While the serum FSH level has a good inverse correlation with decreased ovarian reserve, this might not true for the ovaries that have a good ovarian reserve. Setting the threshold at 140 for MORPI and at 0.95 for ORPI, we could obtain the optimal compromise between specificity and sensitivity.

The ROC curves also revealed good prognostic potency for all other factors (Age, AMH and AFC) analysed. However, the AUC presented by the MORPI and ORPI were always higher than Finally regarding the pregnancy occurrence (Table 3 and Figure 5) ROC curve showed an area under the curve of 0.708 for MORPI while it was 0.702 for ORPI indicating that both MORPI and ORPI have good prognostic potency. Setting the threshold at 63.5 for MORPI, offered the optimal compromise between specificity and sensitivity. At a cut-off level of 0.365 for ORPI, we got optimal compromise between sensitivity and specificity. ROC curves also revealed good prognostic potency for all other factors (age, AMH and AFC) analysed. However, the AUC presented by the MORPI was always higher than those presented by all other factors.

\section{DISCUSSION}

In spite of many advances in the field of reproductive medicine, the risk of poor ovarian response and excessive ovarian response following $\mathrm{COS}$, have remained as a strong obstacle in many programs. A reliable indicator for providing more precise estimates of the patients' ovarian response might facilitate the optimization and individualization of controlled ovarian stimulation (COS) protocol before the onset of a treatment cycle. The European Society of Human Reproduction and Embryology Consensus Conference has established a standardized definition of poor ovarian response as theretrieval of $<4$ oocytes following a standard IVF protocol. ${ }^{4}$ Similar to poor response, prediction of excessive response is also of great importance for the counseling and management of infertile women in IVF clinical practice. The "high response" is generally termed as the retrieval of $>15$ oocytesfollowing a standard COS protocol. ${ }^{5,6}$ There is no definite rule and extremes of response may occur unexpectedly. For example women with PCOS are known to be at high risk of OHSS. However, these patients constitute only $20 \%$ of subjects undergoing COS and less than one fifth of those will present with symptoms of OHSS. Therefore, there is a real need for finding predictive factors that can be used in daily clinical practice to improve the whole patient's IVF experience and to predict the individual stimulation regimen.

The present study proposes a new index, the Modified Ovarian Response Index (MORPI), to identify the probable ovarian response to stimulation during the ART cycles. The combination of different variables in the MORPI resulted in a more precise index to predict the ovarian response. We also compared this new index with other markers of ovarian reserve and another index, the ORPI (ovarian response prediction index), developed by Oliveira et al. ${ }^{3}$ As described previously ORPI takes into account three factors of ovarian reserve namely the age (years) of the patient, the number of antral follicles and the AMH (ng/ml) level. In addition to above three factors we incorporate serum FSH level of the patient to develop the new index, MORPI. Indeed, the results showed significant correlations $(\mathrm{P}<0.001)$ between the MORPI values and the number of obtained follicles and the number and maturity of the collected oocytes. In addition, the results using the MORPI were always better than those results obtained using other predictive factors (AFC, AMH, FSH and age) separately. These findings support the use of this simple 4-variable index.

A woman's age is considered as a prognostic factor for infertile couples undergoing IVF. A marked decline in success rates is observed after 35 years of age. Because pituitary FSH secretion increases with declining ovarian reserve, day 3 serum FSH concentrations are being routinely used in most ART programs and this has been found to be a better predictor of ART outcome than age. ${ }^{7}$

The age of the patient shows a good correlation with the ovarian response. This could be partially due to the link between age and ovarian reserve (AMH and AFC), as previously described by Cui et al. ${ }^{8}$ However, age appeared to have an additional predictive value in some studies for high as well as for low response. Basal serum FSH levels are inversely linked to the ovarian response, mainly by their correlation with $\mathrm{AMH}$ and AFC. However, the polymorphisms of FSH receptors also suggest the fact that when FSH levels increase there is a need for higher doses of gonadotropins for stimulation. ${ }^{9,11}$

For poor responders, Burks et al, have shown that an AMH lower than $0.5 \mathrm{ng} / \mathrm{ml}$ permitted discrimination between successful and unsuccessful stimulation with $83 \%$ sensitivity and $71 \%$ specificity. ${ }^{12}$ In our study we found An AMH cut-off of $1.4 \mathrm{ng} / \mathrm{ml}$ for collecting <4 oocytes. Using a receiver operating characteristics (ROC) curve, Oehninger et al, also shown AFC has the ability to predict a high ovarian response (area under the curve $($ AUC $)=0.882)$ as a low response $($ AUC $=0.882) .{ }^{9}$ Nelson et al, found a better predictive value of $\mathrm{AMH}$ versus AFC for oocyte yield. ${ }^{13}$ In contrast we found a better predictive value of AFC than AMH for retrieval of $\geq 4$ oocytes, however for retrieval of $\geq 4$ M-II oocytes, we found a better predictive value of AMH than AFC. 
It should be noted that in the Nelson study, 19 assisted reproductive technology centres participated. Because AFC has been shown to have important inter observer variations, this discrepancy could be explained by the fact that our study was performed in a single centre with only a single operator. ${ }^{14}$ Previously, anomogram based on age, AMH and day 3 FSH was proposed to predict the FSH starting dose before an agonist protocol, but this only explained $30 \%$ of the variability in ovarian sensitivity ( $\mathrm{r}$ $=0.31) .{ }^{15}$ Nevertheless, a randomized trial of the use of this nomogram for agonist protocols allowed an optimal response of $63 \%$ to be obtained versus $42 \%$ in the control group. ${ }^{16}$

For collection of $\geq 4$ oocytes, we found a cut-off of 0.25 (AUC-0.921) for ORPI and 35 (AUC-0.952) for MORPI. Similarly, Oliveira et al, found a cut-off value of 0.2 (AUC- 0.91) for ORPI. ${ }^{3}$ As far as collection of $\geq 4 \mathrm{M}-\mathrm{II}$ oocytes is concerned at a cut-off value of 0.475 (AUC0.943) for ORPI and 108.33 (AUC-0.953) for MORPI, we obtained optimum sensitivity and specificity. Similarly, Oliveira et al, found a cut-off value of 0.3 (AUC- 0.84) for ORPI. ${ }^{3}$

On the other hand for collection of $\geq 15$ oocytes, we found a cut-off of 0.95 (AUC-0.928) for ORPI and 140 (AUC-0.918) for MORPI. Similarly, Oliveira et al, found a cut-off value of 0.9 (AUC- 0.89) for ORPI. ${ }^{3}$ The above observation shows that MORPI is an excellent index and is better than ORPI for determining poor ovarian response like collection of $\geq 4$ oocytes and collection of $\geq 4 \mathrm{M}$-II oocytes. However for determining excessive response, although both MORPI and ORPI, have got excellent performance under ROC curve, ORPI has a slight better predictive value.

Coming to the clinical pregnancy rate, we found a cut-off of 0.365 (AUC-0.702) for ORPI and 63.5 (AUC-0.708) for MORPI. Oliveira et al, found a cut-off value of 0.3 (AUC- 0.74) for ORPI. ${ }^{3}$

Potential limitations of the study was less number of study population and retrospective method of study design are the only relative limitations of this study.

\section{CONCLUSION}

To summarize, the present study describes a new index the MORPI, which is a simple, precise and cost effective index to predict a low ovarian response, the collection of $>4$ MII oocytes and an excessive ovarian response in infertile women. This index also has a good ability to predict the clinical pregnancy rate. This might be used to improve the cost-benefit ratio of ovarian stimulation regimens by guiding the selection of medications and by tailoring the doses and regimens to the actual needs of patients.

Also it can help in counseling patients regarding their cycle cancellation rate, development of OHSS and the pregnancy rate at the beginning of their IVF cycle. However future research is needed to further define the role of MORPI in IVF outcome).

Funding: No funding sources Conflict of interest: None declared

Ethical approval: The study was approved by the Institutional Ethics Committee

\section{REFERENCES}

1. Coccia ME, Rizzello F. Ovarian reserve. Ann N Y AcadSci. 2008;1127:27-30.

2. Sills ES, Alper MM, Walsh AP. Ovarian reserve screening in infertility: practical applications and theoretical directions for research. Eur J Obstet Gynecol Reprod Biol. 2009;146:30-6.

3. Oliveira JBA, Baruffi RL, Petersen CG. A new ovarian response prediction index (ORPI): implications for individualized controlled ovarian stimulation. Reproduct Bio Endocrinol. 2012;10:94.

4. Ferraretti AP, La Marca A, Fauser BC. ESHRE consensus on the definition of 'poor response' to ovarian stimulation for in vitro fertilization: the Bologna criteria. Hum Reprod. 2011;26:1616-24.

5. La Marca A, Sighinolfi G, Radi D. Anti-Mullerian hormone $(\mathrm{AMH})$ as a predictive marker in assisted reproductive technology (ART). Hum Reprod Update. 2010;16:113-30.

6. Broer SL, Dolleman M, Opmeer BC. AMH and AFC as predictors of excessive response in controlled ovarian hyper stimulation: a meta analysis. Hum Reprod Update. 2011;17:46-54.

7. Karimzadeh M, Ghandi S. Age and basal FSH as a predictor of ART outcome. Iranian J Reproduct Med. 2009; 7(1):19-22.

8. Cui Y, Shi Y, Cui L, Han T, Gao X, Chen ZJ. Agespecific serum antimullerian hormone levels in women with and without polycystic ovary syndrome. Fertil Steril. 2014;102(1):230-236.e2.

9. Oehninger S, Nelson SM, Verweij P, Stegmann BJ. Predictive factors for ovarian response in a corifollitropinalfa/GnRH antagonist protocol for controlled ovarian stimulation in IVF/ICSI cycles. Reprod Biol Endocrinol. 2015;13:117.

10. Desai SS, Achrekar SK, Paranjape SR, Desai SK, Mangoli VS, Mahale SD. Association of allelic combinations of FSHR genepolymorphisms with ovarian response. Reprod Biomed. 2013;27(4):400-6.

11. Lazaros L, Hatzi E, Xita N. Influence of FSHR diplotypes on ovarian response to standard gonadotropin stimulation for IVF/ICSI. J Reprod Med. 2013;58:395-401.

12. Burks HR, Ross L, Opper N, Paulson E, Stanczyk FZ, Chung K. Can highly sensitive anti müllerian hormone testing predict failed response to ovarian stimulation? Fertil Steril. 2015;104(3):643-8.

13. Nelson S, Klein B, Arce J. Comparison of antimüllerian hormone levels and antral follicle count as predictor of ovarian response to controlled 
ovarian stimulation in good-prognosis patients at individual fertility clinics in two multicenter trials. Fertil Steril. 2015;103(4):923-930.e1.

14. Iliodromiti S, Kelsey TW, Wu O, Anderson RA, Nelson S. The predictive accuracy of anti-Müllerian hormone for live birth after assisted conception: a systematic review and meta-analysis of the literature. Hum Reprod Update. 2014;20(4):560-70.

15. La Marca A, Papaleo E, Grisendi V, Argento C, Giulini S, Volpe A. Development of a nomogram based on markers of ovarian reserve for the individualization of the follicle-stimulating hormone starting dose in in vitro fertilization cycles. BJOG. 2012;119(10):1171-9.
16. Allegra A, Marino A, Volpes A, Coffaro F, Scaglione P, Gullo S, et al. A randomized controlled trial investigating the use of a predictive nomogram for the selection of the FSH starting dose in IVF/ICSI cycles. Reprod Biomed. 2017;34(4):42938.

Cite this article as: Kalpana B, Panda SR. Modified ovarian response prediction index: a novel index for ovarian response prediction in GnRH agonist cycles. Int J Reprod Contracept Obstet Gynecol 2019;8:2575-81. 